\title{
Spatial and Temporal Drought Analysis in Sitanadi Basin, Udupi District Karnataka, using SPI
}

\author{
V. Anantharama, K. Gajalakshmi, M. Lokeshwari
}

\begin{abstract}
Knowledge of trends and variations of current and historical hydro-climatological variables are pertinent to the future development and sustainable management of water resources of a given region. Rainfall is a principal element of the hydrological cycle, so that understanding its behavior may be of profound social and economic significance. The detection of trends and oscillations in precipitation time series yields important information for the understanding of climate. However, rainfall changes are particularly hard to gauge, because rainfall is not uniform and varies considerably from place to place and time to time, even on small scales. The Sitanadi basin has a tropical monsoon climate. The highland of the district experiences mainly orographic rainfall. Rainfall data for 42 years were considered for the study and SPI was calculated for the whole period. SPI values of the rain gauge stations of the Sitanadi basin were found to vary from +2.43 to -2.50 . In all rain gauge stations higher abnormality is observed in pre monsoon season compared to post monsoon season. The validation based on station gauge rainfall data showed that the SPIs calculated were reliable for monitoring drought regions.
\end{abstract}

Index Terms: Rainfall, Sitanadi basin, Standardized precipitation index

\section{INTRODUCTION}

Hydrometeorology is concerned with the study of the atmosphere and land phases of the hydrological cycle with emphasis on the inter-relationships involved. This not only involves the atmospheric stages of the hydrological cycle but also part of what is normally ascribed to surface water hydrology. Meteorology deals with the atmospheric portion of the cycle and is often defined as the science of atmospheric phenomena.

Several studies have been carried out at different temporal scales and in different parts of the globe. Existing analyses of daily series show for some areas a positive trend in the daily precipitation intensity and a tendency toward higher frequencies of heavy and extreme rainfall in the last few decades (Houghton et al., 1996). Over India sub-continent, rainfall analysis between 1871 and 1994 indicated decreasing trends during 1880-1905 and 1945-1965 with increasing trends at other periods (Naidu et al., 1999). Similar studies

Revised Manuscript Received on July 22, 2019

Dr. V. Anantharama, Associate Professor, R.V College of Engineering, Bengaluru, India - 560059

Dr. K. Gajalakshmi, Assistant Professor, Department of Civil Engineering. R.V College of Engineering, Bengaluru, India - 560059.

Dr. M. Lokeshwari, Assistant Professor, Department of Civil Engineering, R.V College of Engineering, Bengaluru, India - 560059. over India reveal that there are significant differences in rainfall trends at the regional level (Guhathakurta and Rajeevan, 2007; Krishinakumar, et. al., 2009). Therefore, there is a need for continuous rainfall studies for the purpose of long-term water resources planning and management. The main objective of these analyses was to examine trends, variability and oscillations in rainfall series of Sitanadi basin.

\section{STUDY AREA}

Sitanadi basin is located between 1320' and 1335' N latitudes and 7440' and 7510' E longitude in Figure. 1 with geographical area of the basin is about $643.65 \mathrm{sq} \mathrm{km}$. Sitanadi basin is placed in Udupi distinct of Karnataka, west coast of peninsular India. In Sitanadi basin, physiographical divisions consist of low land, mid land and high land. It has small lateritic ridges with cultivable low lands, in between small exposures of gneisses and laterite hillocks with sparse vegetation. The midland region consists of laterite ridges, mesas and also structural hills composed of gneisses with incised narrow valleys of younger cycle. Average maximum and minimum humidity, pan evaporation, actual sun shine and wind velocity are $96.53 \%$ in July, $41.12 \%$ in January, $6.09 \mathrm{~mm} /$ day in April, 9.62 hours/day in February and 3.64 $\mathrm{Km} / \mathrm{h}$ in April respectively.

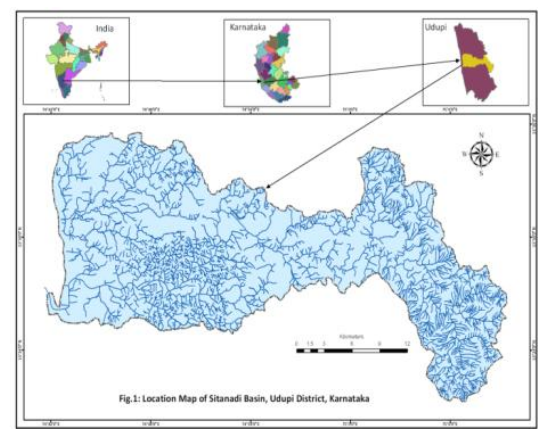

The maximum temperature recorded during April is about $35^{\circ} \mathrm{C}$ and the minimum temperature of $18.54^{\circ} \mathrm{C}$ in January. The rainfall in the basin is mainly due to convection and orographic. It receives an approximate average rainfall of $4832 \mathrm{~mm}$ and about $90 \%$ of which occurs during the monsoon period (June-September).

In the study area about $32 \%$ of the land is covered by tree groves, $28 \%$ area are covered by forest land and $25 \%$ of the area is covered by agricultural land. The convective precipitation, typical of the

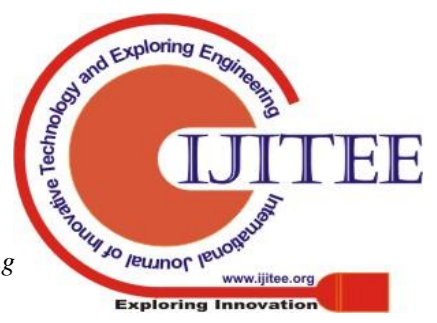


tropics, is caused by the heating of air at the interface with the ground and is characterized by light showers or storms of extremely high intensity. The convective storms experienced by the Karnataka coast are usually local in nature and often result in very intense rainfall. Orographic rainfall in the district is the result of mechanical lifting of moist horizontal air currents over the Western Ghats region.

\section{RAINFALL ANALYSIS}

In the study area, there are six rain gauging stations situated in and around the basin. Rainfall data from these stations for 1976 - 2017 (42 years) period were collected from District Statistical officer and Dept of Economics and Statistics, GOK, Bangalore. The monthly average rainfalls in different stations are shown in Table.1.

\section{A. Monthly Rainfall Analysis}

In case of monthly rainfall, mean rainfall during July (Table.5) is the highest is $1518.98 \mathrm{~mm}$ and contributes to $31.90 \%$ of annual rainfall followed by June $1196.13 \mathrm{~mm}$ (25.12\%). Monthly mean rainfall for July is highest (1518.98 $\mathrm{mm}$, Table.5) and contributes $31.9 \%$ of annual rainfall. This is followed by June month that contributes $25.12 \%$ of annual rainfall $(1196.13 \mathrm{~mm})$. During August $(1169 \mathrm{~mm})$ and September $(405.22 \mathrm{~mm})$ months contributes $24.55 \%$ and $8.51 \%$ of the annual rainfall respectively. Rainfall in January is the least with $10.26 \mathrm{~mm}$ and that contributes only $0.22 \%$ to the annual rainfall. From October to December and February to May contributes about $220.83 \mathrm{~mm}(4.64 \%), 84.90 \mathrm{~mm}$ (1.78\%), $39.01 \mathrm{~mm}(0.82), 10.88 \mathrm{~mm}(0.23 \%), 31.63 \mathrm{~mm}$ $(0.66 \%), 37.63 \mathrm{~mm}(0.79)$, and $171.63 \mathrm{~mm}(3.60 \%)$ of rainfall respectively. The coefficient of variation is highest during December $(258.98 \%)$ and is followed by March (202.57\%), January (120.79\%), April (101.15\%) and May $(97.61 \%)$. Low coefficient of variation during the high rainfall months of June (35.47\%) and July (42.66\%).

\section{B. Depth of rainfall over the basin}

In order to understand the areal distribution of rainfall from the existing rainfall stations in Sitanadi basin, methods such as unweight arithmetic mean, Thiessen polygon and isohyet approach are made use of. The average depth of rainfall over the basin by these three methods is given in Table.3. Thiessen polygon and the isohyet maps prepared for the basin are shown in Fig 2 and 3.

Table.1 Depth of rainfall over the basin

\begin{tabular}{cc}
\multicolumn{2}{c}{ Table.1 Depth of rainfall over the basin } \\
\hline Method & Depth of rainfall $(\mathbf{m m})$ \\
\hline Arithmetic mean & 4923 \\
Thiessen polygon & 4832 \\
Isohyet & 4759 \\
\hline
\end{tabular}

\section{Statistical Analysis of Rainfall}

Precipitation ratio (PR) is the ratio between the range (difference between maximum and minimum rainfall) and mean of rainfall of respective year expressed in percentage. The abnormalities of rainfall at any location may be brought by a simple ratio of precipitation. This ratio may give the stability of rainfall with special relationship. Higher the PR higher in abnormality in rainfall and vice versa (Rathod \& Aruchamy, 2010). Coefficient of variation is the ratio of standard deviation to mean expressed in percentage. Variability defined as the deviation from mean or ratio of the standard deviation to the mean rainfall and in other words variability of coefficient of variation $(\mathrm{CV})$. It indicates the consistency of rainfall in a particular period. If the value of $\mathrm{CV}$ is low, it indicates the rainfall is stable at that particular period and vice-versa.

Table.3 Monthly average rainfall in rain gauge stations of Sitanadi basin (1976 - 2017)

\begin{tabular}{cccccccc}
\hline $\begin{array}{c}\text { Mont } \\
\text { h }\end{array}$ & $\begin{array}{c}\text { Brahm } \\
\text { avara }\end{array}$ & Kota & Haladi & Ardi & $\begin{array}{c}\text { Sitana } \\
\text { di }\end{array}$ & $\begin{array}{c}\text { Agum } \\
\text { be }\end{array}$ & Average \\
\hline Jan & 8.2 & 4.3 & 29.8 & 4.1 & 17.8 & 5.0 & $\mathbf{1 1 . 5}$ \\
Feb & 5.8 & 7.0 & 11.3 & 19.0 & 9.0 & 9.6 & $\mathbf{1 0 . 3}$ \\
Mar & 40.3 & 61.0 & 23.1 & 67.8 & 43.8 & 8.6 & $\mathbf{4 0 . 7}$ \\
Apr & 25.9 & 32.2 & 40.1 & 48.9 & 52.8 & 37.0 & $\mathbf{3 9 . 5}$ \\
May & 191.3 & 154.4 & 132.0 & 166.4 & 192.6 & 181.6 & $\mathbf{1 6 9 . 7}$ \\
Jun & 1030.1 & 982.4 & 1074.3 & 1139. & 1231.5 & 1827.5 & $\mathbf{1 2 1 4 . 1}$ \\
Jly & 1162.5 & 1092.2 & 1419.0 & $\begin{array}{c}1537 . \\
8\end{array}$ & 1769.6 & 2453.3 & $\mathbf{1 5 7 2 . 4}$ \\
Aug & 846.2 & 769.8 & 1178.8 & 1133. & 1395.3 & 2049.5 & $\mathbf{1 2 2 8 . 8}$ \\
Sep & 328.7 & 343.5 & 398.8 & 433.4 & 464.3 & 565.4 & $\mathbf{4 2 2 . 4}$ \\
Oct & 185.3 & 165.1 & 189.9 & 275.3 & 278.2 & 276.8 & $\mathbf{2 2 8 . 4}$ \\
Nov & 76.7 & 67.3 & 85.9 & 118.3 & 105.1 & 64.1 & $\mathbf{8 6 . 2}$ \\
Dec & 24.3 & 26.4 & 31.0 & 47.2 & 37.4 & 231.4 & $\mathbf{6 6 . 3}$ \\
\hline Total & $\mathbf{3 9 2 5 . 4}$ & $\mathbf{3 7 0 5 . 5}$ & $\mathbf{4 6 1 4 . 0}$ & $\mathbf{4 9 9 0 .}$ & $\mathbf{5 5 9 7 . 4}$ & 7709.7 & \\
\hline & & & & & & & \\
\hline
\end{tabular}

\section{Statistical Analysis of Monthly and Yearly Rainfall}

The average coefficient of variation of annual rainfall is $18.73 \%$ (Table.4). This indicates that it is highly stable (Krishnakumar et al, 2009). In case of monthly rainfall, mean rainfall during July (Table.5) is the highest is $1518.98 \mathrm{~mm}$ and contributes to $31.90 \%$ of annual rainfall followed by June $1196.13 \mathrm{~mm}$ (25.12\%). Monthly mean rainfall for July is highest (1518.98 mm, Table.5) and contributes $31.9 \%$ of annual rainfall. This is followed by June month that contributes $25.12 \%$ of annual rainfall $(1196.13 \mathrm{~mm})$. During August $(1169 \mathrm{~mm})$ and September $(405.22 \mathrm{~mm})$ months contributes $24.55 \%$ and $8.51 \%$ of the annual rainfall respectively. Rainfall in January is the least with $10.26 \mathrm{~mm}$ and that contributes only $0.22 \%$ to the annual rainfall. From October to December and February to May contributes about $220.83 \mathrm{~mm}(4.64 \%), 84.90 \mathrm{~mm}(1.78 \%), 39.01 \mathrm{~mm}(0.82)$, $10.88 \mathrm{~mm}(0.23 \%), 31.63 \mathrm{~mm}(0.66 \%), 37.63 \mathrm{~mm}(0.79)$, and $171.63 \mathrm{~mm}(3.60 \%)$ of rainfall respectively. The coefficient of variation is highest during December (258.98\%) and is followed by March (202.57\%), January (120.79\%), April $(101.15 \%)$ and May $(97.61 \%)$. Low coefficient of variation during the high rainfall months of June $(35.47 \%)$ and July $(42.66 \%)$

The same trend is observed in case of precipitation ratio also. Rain fall in the pre monsoon season (March to May) and part of post monsoon (January and February) is very unstable compared to the other part of the year. Monsoon season rainfall is highly stable. The lower PR is observed (60.33) in Haladi rain gauge station and higher PR is observed (75.20) in Agumbe rain gauge station. Based on the PR it can be concluded that the rainfall is very stable in the Sitanadi basin because the variation of PR between rain gauge stations is found very small (Table.4).

Published By: 


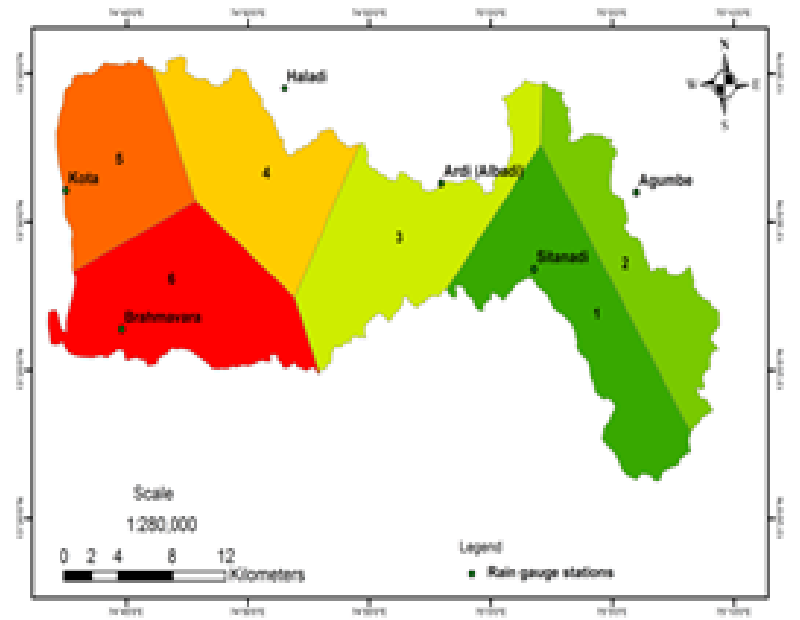

Fig.2. Thiessen Polygon for Sitanadi basin

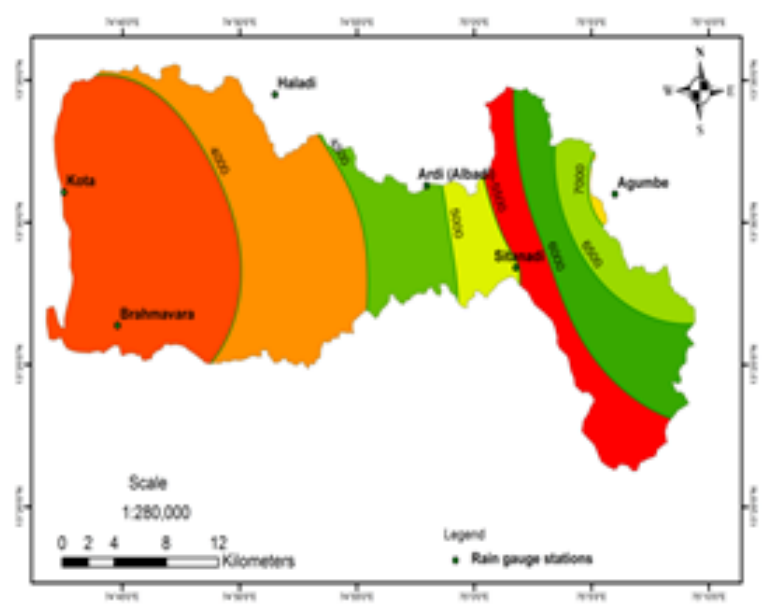

Fig.3. Isohyetal map of Sitanadi basin

\section{E. Statistical Analysis of Seasonal Rainfall}

Season wise rainfall statistics are shown in Table.6. In Sitanadi basin PR varies from 67.61 at Agumbe to 2283.61 at Haladi rain gauge station during monsoon season. PR varies between 184.98 (Ardi) to 364.62 (Agumbe) in POM season and in PRM season PR varies between 313.25 (Sitanadi) and 510.3 (Agumbe). In monsoon season all rain gauge stations except Haladi and Ardi shows lower PR. In POM and PRM seasons all rain gauge stations shows a moderate PR. PR values indicate that higher abnormality of rainfall is observed in Haladi and Ardi rain gauge stations in monsoon season. Average annual rainfall during the southwest monsoon (June-September) is about $89.86 \%$. It varies from $87.72 \%$ in Ardi to $92.73 \%$ in Agumbe (Table.7) of the annual rainfall. The average contribution of POM (Oct-Jan) and PRM (Feb-May) rainfall to the annual is $6.17 \%$. It varies from $4.72 \%$ at Ardi to $6.87 \%$ at Sitanadi during POM and in PRM it is $3.83 \%$. During PRM it varies between $2.95 \%$ at Haladi to $4.97 \%$ at Barahmavara. From the Table.7, the seasonal rainfall during monsoon is dependable because the coefficient of variation is $19.30 \%$. At the same time, rainfall during POM (257.94\%) and PRM (338.18\%) are undependable as the coefficient of variation is very high.

\section{F. Variation in Decade-Wise Annual and Seasonal Rainfall}

Year-wise percentage annual rainfall frequencies of excess and deficit years are depicted in Table.8. During the period from 1976-77 to 1978-79 there were 3, 2, 2, 2 excessive rainfall years in Brahmavara, Kota, Haladi and Ardi rain gauge stations and deficit rainfall is not noticed in any of the stations. During 1970-71 to 1979-80, there were 3, 1,2 , and 3 excessive rainfall years in Brahmavara, Ardi, Sitanadi, and Agumbe respectively and 2, 2, 2 and 2 years of deficit rainfall years in Haladi, Ardi, Sitanadi and Agumbe respectively is observed. In the decade from 1991-92 to 1999-2000, there were 2 and 1 excessive rainfall years in Kota and Agumbe respectively and there were 4, 2 and 1 deficit rainfall years is noticed in Brahmavara, Kota and Sitanadi respectively. In the next decade, between 2001-2002 and 2010-2017 there were 1, 2, 1, 1, 2, and 4 excessive rainfall years in Brahmavara, Ardi, Kota, Haladi, Sitanadi and Agumbe respectively and there were one deficit rainfall year in Ardi and Agumbe. In the decade between 2007-08 and 2016-17 there were 1, 2, 2, and 1 excessive rain fall years in Kota, Haladi, Ardi, and Sitanadi respectively. Deficit numbers of rainfall years were 2, 2, 1, 2 and 5 in Brahmavara, Haladi, Ardi, Sitanadi and Agumbe rain gauge stations respectively. Excessive percentage rainfall years goes on reducing from the year 1980-81 to2009-2017 and slight increase is observed in the decade from 2011-2017 (Table 3.8). Regarding percentage of normal rainfall years, it increased from the year 1980-81 up to 2009-2010. In the last decade, from the year 2009-2017 there is a drop in the percentage of normal rainfall years. Except the rain gauge stations at Kota and Ardi all other stations shows increase in the percent of deficit rainfall years in the last decade between 2009-2017. During the period between 2009 and 2017, it is found that in Brahmavara, Haladi and Sitanadi, there are about $22 \%$ deficit rainfall years and in Agumbe it is $56 \%$ deficit rainfall years.

\section{G. Variation in Decade Wise Monthly Rainfall Contribution}

Variation of decadal monthly contribution of rainfall is presented in Table 3.9. In the month of June, July and August there is a rise and fall between the decades and does not show any trend in rainfall. In all the rain gauge stations, there is 6 to $7 \%$ increase in rainfall in the month of June during 2001-2009 to 2010-17.

During the same period, there is decrease in rainfall (about $10 \%$ ) in the month of July in all rain gauge stations. In September and October months, increase in rainfall is noticed from 1980-90 to 2010-17 in all the rain gauge stations. All the rain gauge stations show almost a uniform trend in percentage contributions between the decades to decade.

Table.4 Statistical analysis of yearly rainfall

\begin{tabular}{llllllll}
\hline $\begin{array}{l}\text { Rain } \\
\text { gauge } \\
\text { station }\end{array}$ & $\begin{array}{l}\text { Min } \\
(\mathbf{m m})\end{array}$ & $\begin{array}{l}\text { Max } \\
(\mathbf{m m})\end{array}$ & $\begin{array}{l}\text { Rang } \\
\mathbf{( m m})\end{array}$ & $\begin{array}{l}\text { Mean } \\
(\mathbf{m m})\end{array}$ & $\begin{array}{l}\text { SD } \\
(\mathbf{m m})\end{array}$ & $\begin{array}{l}\text { CV } \\
(\boldsymbol{\%})\end{array}$ & PR \\
\hline Brahmava & 2431. & 5597.6 & 3166. & 3786. & 744.8 & 19.6 & 64.2 \\
ra & 14 & 4 & 50 & 76 & 9 & 7 & 0 \\
Kota & 2235. & 4504.2 & 2268. & 3575. & 546.2 & 15.2 & 62.5
\end{tabular}




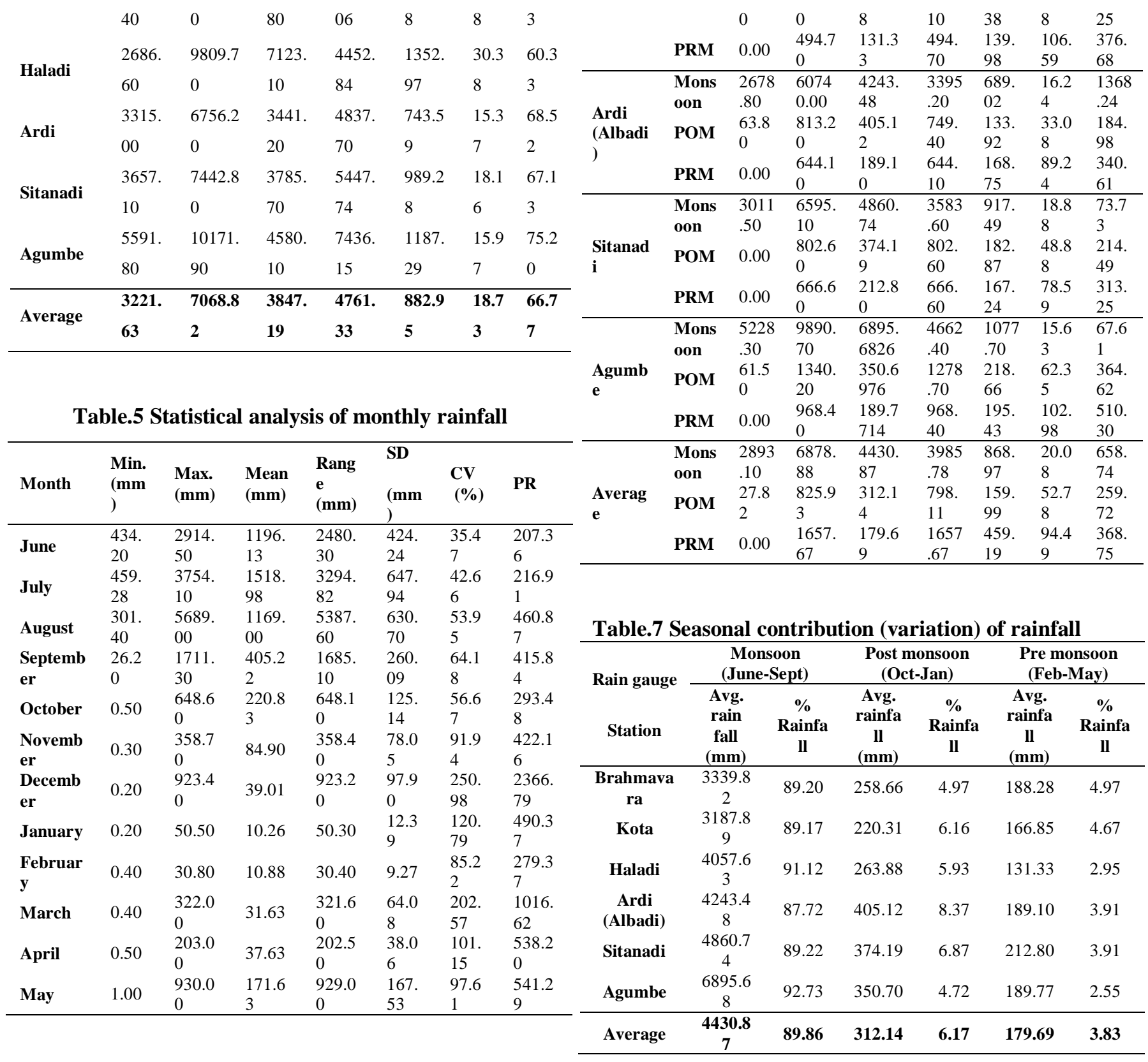

Table.6 Season wise rainfall statistics

\begin{tabular}{lllllllll}
\hline $\begin{array}{l}\text { Station } \\
\text { S }\end{array}$ & $\begin{array}{l}\text { Seaso } \\
\text { n }\end{array}$ & Min & Max & Mean & $\begin{array}{l}\text { Ran } \\
\text { ge }\end{array}$ & SD & $\begin{array}{l}\text { CV }( \\
\text { \%) }\end{array}$ & PR \\
\hline & Mons & 2017 & 4980. & 3339. & 2963 & 697. & 20.9 & 88.7 \\
Brahm & oon & .40 & 79 & 81 & .39 & 50 & 0 & 3 \\
avara & POM & 11.9 & 654.2 & 258.6 & 642. & 144. & 55.7 & 248. \\
& & 3 & 0 & 6 & 27 & 28 & 8 & 31 \\
& PRM & 00.0 & 6640. & 188.2 & 6640 & 1940 & 103. & 352. \\
& Mons & 1972 & 4221. & 3187. & 2248 & 543. & 17.0 & 70.5 \\
& oon & .90 & 70 & 89 & .80 & 88 & 6 & 4 \\
& POM & 0.00 & 631.6 & 220.3 & 631. & 138. & 63.0 & 286. \\
& & & 0 & 1 & 60 & 83 & 1 & 69 \\
& PRM & 0.00 & 532.2 & 166.8 & 532. & 143. & 86.1 & 318. \\
& & & 0 & 5 & 20 & 72 & 4 & 97 \\
\hline \multirow{3}{*}{ Haladi } & Mons & 2449 & 9511. & 4057. & 7061 & 1288 & 31.7 & 2283 \\
& oon & .70 & 00 & 63 & .30 & .21 & 5 & .61 \\
& POM & 29.7 & 713.8 & 263.8 & 684. & 141. & 53.5 & 259.
\end{tabular}

Table.8 Decadal variation (Excess, deficit and normal) of rainfall

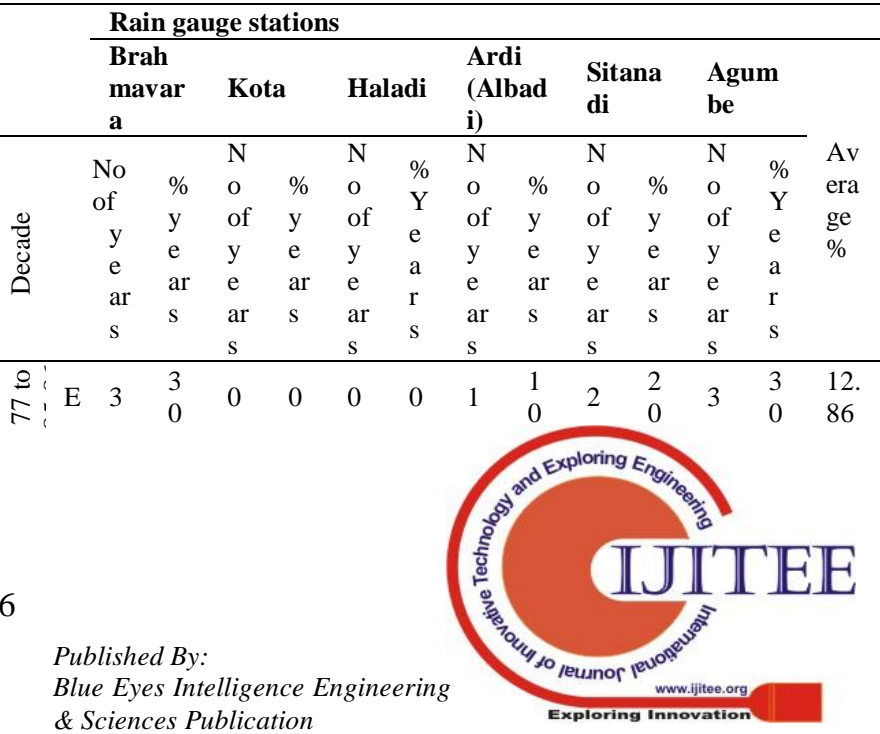




\begin{tabular}{|c|c|c|c|c|c|c|c|c|c|c|c|c|c|c|}
\hline & D & 0 & 0 & 0 & 0 & 2 & $\begin{array}{l}2 \\
0\end{array}$ & 2 & $\begin{array}{l}2 \\
0\end{array}$ & 2 & & 2 & $\begin{array}{l}2 \\
0\end{array}$ & $\begin{array}{l}12 . \\
86\end{array}$ \\
\hline & $\mathrm{N}$ & 7 & $\begin{array}{l}7 \\
0\end{array}$ & $\begin{array}{l}1 \\
0\end{array}$ & $\begin{array}{l}1 \\
0 \\
0\end{array}$ & 8 & $\begin{array}{l}8 \\
0\end{array}$ & 7 & $\begin{array}{l}7 \\
0\end{array}$ & 6 & $\begin{array}{l}6 \\
0\end{array}$ & 5 & $\begin{array}{l}5 \\
0\end{array}$ & $\begin{array}{l}74 . \\
29\end{array}$ \\
\hline ڤ & E & 0 & 0 & 3 & 0 & 0 & 0 & 0 & 0 & 1 & $\begin{array}{l}1 \\
0\end{array}$ & 1 & $\begin{array}{l}1 \\
0\end{array}$ & $\begin{array}{l}5.7 \\
1\end{array}$ \\
\hline $\begin{array}{l}1 \\
\alpha \\
o\end{array}$ & D & 4 & $\begin{array}{l}4 \\
0\end{array}$ & 2 & $\begin{array}{l}2 \\
0\end{array}$ & 0 & 0 & 0 & 0 & 1 & $\begin{array}{l}1 \\
0\end{array}$ & 0 & 0 & $\begin{array}{l}10 . \\
00\end{array}$ \\
\hline $\begin{array}{l}0 \\
0 \\
0 \\
0 \\
0 \\
\varrho\end{array}$ & $\mathrm{N}$ & 6 & $\begin{array}{l}6 \\
0\end{array}$ & 5 & $\begin{array}{l}5 \\
0\end{array}$ & $\begin{array}{l}1 \\
0\end{array}$ & $\begin{array}{l}1 \\
0 \\
0\end{array}$ & $\begin{array}{l}1 \\
0\end{array}$ & $\begin{array}{l}1 \\
0 \\
0\end{array}$ & 8 & $\begin{array}{l}8 \\
0\end{array}$ & 9 & $\begin{array}{l}9 \\
0\end{array}$ & $\begin{array}{l}80 . \\
00\end{array}$ \\
\hline $2 \%$ & $\mathrm{E}$ & 1 & $\begin{array}{l}1 \\
0\end{array}$ & 2 & $\begin{array}{l}2 \\
0\end{array}$ & 1 & 0 & 1 & 0 & 2 & 0 & 4 & 0 & $\begin{array}{l}4.2 \\
9\end{array}$ \\
\hline 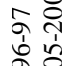 & D & 0 & 0 & 0 & 0 & 0 & 0 & 0 & 0 & 0 & 0 & 1 & $\begin{array}{l}1 \\
0\end{array}$ & $\begin{array}{l}2.8 \\
6\end{array}$ \\
\hline ડે & $\mathrm{N}$ & 9 & $\begin{array}{l}9 \\
0\end{array}$ & 8 & $\begin{array}{l}8 \\
0\end{array}$ & 9 & $\begin{array}{l}9 \\
0\end{array}$ & 9 & $\begin{array}{l}9 \\
0\end{array}$ & 8 & $\begin{array}{l}8 \\
0\end{array}$ & 5 & $\begin{array}{l}5 \\
0\end{array}$ & $\begin{array}{l}81 . \\
43\end{array}$ \\
\hline$\frac{1}{b}$ & E & 0 & 0 & 1 & $\begin{array}{l}1 \\
2\end{array}$ & 2 & $\begin{array}{l}2 \\
2\end{array}$ & 2 & $\begin{array}{l}2 \\
2\end{array}$ & 1 & $\begin{array}{l}1 \\
2\end{array}$ & 0 & 0 & $\begin{array}{l}9.7 \\
1\end{array}$ \\
\hline$\stackrel{1}{0}$ & $\mathrm{D}$ & 2 & $\begin{array}{l}2 \\
2\end{array}$ & 0 & 0 & 2 & $\begin{array}{l}2 \\
2\end{array}$ & 1 & $\begin{array}{l}1 \\
2\end{array}$ & 2 & $\begin{array}{l}2 \\
2\end{array}$ & 5 & $\begin{array}{l}5 \\
6\end{array}$ & $\begin{array}{l}23 . \\
86\end{array}$ \\
\hline ठ̊. & $\mathrm{N}$ & 7 & $\begin{array}{l}7 \\
8\end{array}$ & 8 & $\begin{array}{l}8 \\
8\end{array}$ & 5 & $\begin{array}{l}5 \\
6\end{array}$ & 6 & $\begin{array}{l}6 \\
6\end{array}$ & 6 & $\begin{array}{l}6 \\
6\end{array}$ & 4 & $\begin{array}{l}4 \\
4\end{array}$ & $\begin{array}{l}66 . \\
43\end{array}$ \\
\hline
\end{tabular}

Note: E-Number of excessive rainfall years, D- Number of deficit rainfall years, N-Number of normal rainfall years

Table.9 Decadal monthly percentage rainfall contribution

\begin{tabular}{|c|c|c|c|c|c|c|c|c|c|c|c|c|}
\hline $\begin{array}{l}\text { Mon } \\
\text { th }\end{array}$ & $\begin{array}{l}\mathrm{Ju} \\
\text { ne }\end{array}$ & $\begin{array}{l}\mathrm{Ju} \\
\text { ly }\end{array}$ & $\begin{array}{l}\text { A } \\
\text { ug }\end{array}$ & $\begin{array}{l}\text { Se } \\
\mathrm{p}\end{array}$ & $\begin{array}{l}\mathrm{O} \\
\mathrm{ct}\end{array}$ & $\begin{array}{l}\mathrm{N} \\
\mathrm{O} \\
\mathrm{V}\end{array}$ & $\begin{array}{l}\mathrm{D} \\
\mathrm{e} \\
\mathrm{c}\end{array}$ & $\begin{array}{l}\mathrm{Ja} \\
\mathrm{n}\end{array}$ & $\begin{array}{l}\mathrm{F} \\
\mathrm{e} \\
\mathrm{b}\end{array}$ & $\begin{array}{l}\text { M } \\
\text { ar }\end{array}$ & $\begin{array}{l}\text { A } \\
\mathrm{pr}\end{array}$ & $\begin{array}{l}M \\
a \\
y\end{array}$ \\
\hline $\begin{array}{l}\text { Deca } \\
\text { de }\end{array}$ & \multicolumn{12}{|c|}{ Brahmavara } \\
\hline $\begin{array}{l}1976 \\
-85\end{array}$ & $\begin{array}{l}25 \\
.1 \\
8\end{array}$ & $\begin{array}{l}35 \\
.1 \\
4\end{array}$ & $\begin{array}{l}22 \\
.7 \\
7\end{array}$ & $\begin{array}{l}7 . \\
68\end{array}$ & $\begin{array}{l}3 . \\
8 \\
5\end{array}$ & $\begin{array}{l}1 . \\
5 \\
7\end{array}$ & $\begin{array}{l}0 . \\
1 \\
0\end{array}$ & $\begin{array}{l}0 . \\
0 \\
1\end{array}$ & $\begin{array}{l}0 . \\
0 \\
1\end{array}$ & $\begin{array}{l}0 . \\
0 \\
3\end{array}$ & $\begin{array}{l}0 . \\
4 \\
1\end{array}$ & $\begin{array}{l}3 . \\
4 \\
8\end{array}$ \\
\hline $\begin{array}{l}1986 \\
-95\end{array}$ & $\begin{array}{l}27 \\
.3 \\
2\end{array}$ & $\begin{array}{l}26 \\
.4 \\
2\end{array}$ & $\begin{array}{l}28 \\
.2 \\
5\end{array}$ & $\begin{array}{l}8 . \\
02\end{array}$ & $\begin{array}{l}4 . \\
8 \\
1\end{array}$ & $\begin{array}{l}1 . \\
7 \\
5\end{array}$ & $\begin{array}{l}0 . \\
2 \\
0\end{array}$ & $\begin{array}{l}0 . \\
0 \\
3\end{array}$ & $\begin{array}{l}0 . \\
0 \\
2\end{array}$ & $\begin{array}{l}0 . \\
0 \\
9\end{array}$ & $\begin{array}{l}0 . \\
4 \\
2\end{array}$ & $\begin{array}{l}2 . \\
7 \\
9\end{array}$ \\
\hline $\begin{array}{l}1996 \\
-200 \\
5\end{array}$ & $\begin{array}{l}23 \\
.0 \\
2\end{array}$ & $\begin{array}{l}36 \\
.5 \\
0\end{array}$ & $\begin{array}{l}21 \\
.3 \\
2\end{array}$ & $\begin{array}{l}8 . \\
33\end{array}$ & $\begin{array}{l}4 . \\
9 \\
3\end{array}$ & $\begin{array}{l}1 . \\
8 \\
1\end{array}$ & $\begin{array}{l}0 . \\
7 \\
5\end{array}$ & $\begin{array}{l}0 . \\
0 \\
4\end{array}$ & $\begin{array}{l}0 . \\
0 \\
1\end{array}$ & $\begin{array}{l}0 . \\
0 \\
3\end{array}$ & $\begin{array}{l}0 . \\
4 \\
4\end{array}$ & $\begin{array}{l}2 . \\
8 \\
3\end{array}$ \\
\hline $\begin{array}{l}2006 \\
-201 \\
7\end{array}$ & $\begin{array}{l}29 \\
.1 \\
3 \\
\end{array}$ & $\begin{array}{l}25 \\
.4 \\
8\end{array}$ & $\begin{array}{l}24 \\
.0 \\
0\end{array}$ & $\begin{array}{l}10 \\
.1 \\
6\end{array}$ & $\begin{array}{l}5 . \\
2 \\
6\end{array}$ & $\begin{array}{l}1 . \\
4 \\
3\end{array}$ & $\begin{array}{l}0 . \\
0 \\
8\end{array}$ & $\begin{array}{l}0 . \\
0 \\
2\end{array}$ & $\begin{array}{l}0 . \\
0 \\
4\end{array}$ & $\begin{array}{l}0 . \\
4 \\
1\end{array}$ & $\begin{array}{l}0 . \\
4 \\
5\end{array}$ & $\begin{array}{l}3 . \\
5 \\
5\end{array}$ \\
\hline & \multicolumn{12}{|c|}{ Kota } \\
\hline $\begin{array}{l}1976 \\
-85\end{array}$ & $\begin{array}{l}25 \\
.2 \\
5\end{array}$ & $\begin{array}{l}35 \\
.0 \\
7\end{array}$ & $\begin{array}{l}22 \\
.7 \\
7\end{array}$ & $\begin{array}{l}7 . \\
70\end{array}$ & $\begin{array}{l}3 . \\
8 \\
4\end{array}$ & $\begin{array}{l}1 . \\
5 \\
7\end{array}$ & $\begin{array}{l}0 . \\
1 \\
0\end{array}$ & $\begin{array}{l}0 . \\
0 \\
1\end{array}$ & $\begin{array}{l}0 . \\
0 \\
1\end{array}$ & $\begin{array}{l}0 . \\
0 \\
3\end{array}$ & $\begin{array}{l}0 . \\
4 \\
0\end{array}$ & $\begin{array}{l}3 . \\
4 \\
7\end{array}$ \\
\hline $\begin{array}{l}1986 \\
-95\end{array}$ & $\begin{array}{l}27 \\
.1 \\
9\end{array}$ & $\begin{array}{l}31 \\
.4 \\
4\end{array}$ & $\begin{array}{l}25 \\
.2 \\
9\end{array}$ & $\begin{array}{l}7 . \\
76\end{array}$ & $\begin{array}{l}4 . \\
2 \\
7\end{array}$ & $\begin{array}{l}1 . \\
6 \\
2\end{array}$ & $\begin{array}{l}0 . \\
1 \\
3\end{array}$ & $\begin{array}{l}0 . \\
0 \\
2\end{array}$ & $\begin{array}{l}0 . \\
0 \\
1\end{array}$ & $\begin{array}{l}0 . \\
0 \\
5\end{array}$ & $\begin{array}{l}0 . \\
4 \\
3\end{array}$ & $\begin{array}{l}3 . \\
1 \\
4\end{array}$ \\
\hline $\begin{array}{l}1996 \\
-200 \\
5\end{array}$ & $\begin{array}{l}22 \\
.7 \\
4\end{array}$ & $\begin{array}{l}36 \\
.3 \\
9\end{array}$ & $\begin{array}{l}21 \\
.3 \\
3\end{array}$ & $\begin{array}{l}8 . \\
55\end{array}$ & $\begin{array}{l}4 . \\
9 \\
4\end{array}$ & $\begin{array}{l}1 . \\
8 \\
7\end{array}$ & $\begin{array}{l}0 . \\
7 \\
5\end{array}$ & $\begin{array}{l}0 . \\
0 \\
4\end{array}$ & $\begin{array}{l}0 . \\
0 \\
1\end{array}$ & $\begin{array}{l}0 . \\
0 \\
3\end{array}$ & $\begin{array}{l}0 . \\
4 \\
5\end{array}$ & $\begin{array}{l}2 . \\
9 \\
0\end{array}$ \\
\hline $\begin{array}{l}2006 \\
-201 \\
7\end{array}$ & $\begin{array}{l}28 \\
.7 \\
2\end{array}$ & $\begin{array}{l}25 \\
.6 \\
7\end{array}$ & $\begin{array}{l}23 \\
.8 \\
9\end{array}$ & $\begin{array}{l}10 \\
.3 \\
8\end{array}$ & $\begin{array}{l}5 . \\
2 \\
5\end{array}$ & $\begin{array}{l}1 . \\
4 \\
6\end{array}$ & $\begin{array}{l}0 . \\
0 \\
8\end{array}$ & $\begin{array}{l}0 . \\
0 \\
2\end{array}$ & $\begin{array}{l}0 . \\
0 \\
4\end{array}$ & $\begin{array}{l}0 . \\
4 \\
3\end{array}$ & $\begin{array}{l}0 . \\
4 \\
5\end{array}$ & $\begin{array}{l}3 . \\
6 \\
1\end{array}$ \\
\hline & \multicolumn{12}{|c|}{ Haladi } \\
\hline $\begin{array}{l}1976 \\
-85\end{array}$ & $\begin{array}{l}25 \\
.3 \\
2\end{array}$ & $\begin{array}{l}34 \\
.9 \\
9\end{array}$ & $\begin{array}{l}22 \\
.7 \\
8\end{array}$ & $\begin{array}{l}7 . \\
71\end{array}$ & $\begin{array}{l}3 . \\
8 \\
2\end{array}$ & $\begin{array}{l}1 . \\
5 \\
6\end{array}$ & $\begin{array}{l}0 . \\
1 \\
0\end{array}$ & $\begin{array}{l}0 . \\
0 \\
1\end{array}$ & $\begin{array}{l}0 . \\
0 \\
1\end{array}$ & $\begin{array}{l}0 . \\
0 \\
3\end{array}$ & $\begin{array}{l}0 . \\
4 \\
0\end{array}$ & $\begin{array}{l}3 . \\
4 \\
9\end{array}$ \\
\hline $\begin{array}{l}1986 \\
-95\end{array}$ & $\begin{array}{l}27 \\
.1 \\
2\end{array}$ & $\begin{array}{l}26 \\
.4 \\
5\end{array}$ & $\begin{array}{l}28 \\
.4 \\
7\end{array}$ & $\begin{array}{l}7 . \\
98\end{array}$ & $\begin{array}{l}4 . \\
8 \\
6\end{array}$ & $\begin{array}{l}1 . \\
7 \\
4\end{array}$ & $\begin{array}{l}0 . \\
1 \\
9\end{array}$ & $\begin{array}{l}0 . \\
0 \\
3\end{array}$ & $\begin{array}{l}0 . \\
0 \\
2\end{array}$ & $\begin{array}{l}0 . \\
0 \\
9\end{array}$ & $\begin{array}{l}0 . \\
4 \\
4\end{array}$ & $\begin{array}{l}2 . \\
7 \\
1\end{array}$ \\
\hline
\end{tabular}

$\begin{array}{lllllllllllll}1996 & 22 & 36 & 21 & 8 & 4 . & 1 . & 0 . & 0 . & 0 . & 0 . & 0 . & 3 .\end{array}$ $\begin{array}{lllllllllllll}-200 & .9 & .3 & .2 & 8 . & 9 & 8 & 7 & 0 & 0 & 0 & 4 & 0 \\ 5 & 0 & 4 & 8 & 32 & 9 & 6 & 5 & 4 & 1 & 3 & 8 & 1\end{array}$ $\begin{array}{lllllllllllll}2006 & 28 & 25 & 23 & 10 & 5 . & 1 . & 0 . & 0 . & 0 . & 0 . & 0 . & 3 .\end{array}$ $\begin{array}{lllllllllllll}-201 & .3 & .8 & .8 & .5 & 2 & 5 & 1 & 0 & 0 & 4 & 4 & 6 \\ 7 & 8 & 7 & 3 & 0 & 4 & 0 & 0 & 2 & 4 & 3 & 5 & 4\end{array}$

\begin{tabular}{|c|c|c|c|c|c|c|c|c|c|c|c|c|}
\hline & \multicolumn{12}{|c|}{ Ardi (Albadi) } \\
\hline $\begin{array}{l}1976 \\
-85\end{array}$ & $\begin{array}{l}25 \\
.3 \\
8\end{array}$ & $\begin{array}{l}34 \\
.9 \\
3\end{array}$ & $\begin{array}{l}22 \\
.8 \\
2\end{array}$ & $\begin{array}{l}7 . \\
69\end{array}$ & $\begin{array}{l}3 . \\
8 \\
1\end{array}$ & $\begin{array}{l}1 . \\
5 \\
7\end{array}$ & $\begin{array}{l}0 . \\
1 \\
0\end{array}$ & $\begin{array}{l}0 . \\
0 \\
1\end{array}$ & $\begin{array}{l}0 . \\
0 \\
1\end{array}$ & $\begin{array}{l}0 . \\
0 \\
3\end{array}$ & $\begin{array}{l}0 . \\
3 \\
9\end{array}$ & $\begin{array}{l}3 . \\
4 \\
7\end{array}$ \\
\hline $\begin{array}{l}1986 \\
-95\end{array}$ & $\begin{array}{l}27 \\
.0 \\
0\end{array}$ & $\begin{array}{l}26 \\
.4 \\
3\end{array}$ & $\begin{array}{l}28 \\
.5 \\
3\end{array}$ & $\begin{array}{l}8 . \\
00\end{array}$ & $\begin{array}{l}4 . \\
9 \\
1\end{array}$ & $\begin{array}{l}1 . \\
7 \\
4\end{array}$ & $\begin{array}{l}0 . \\
1 \\
9\end{array}$ & $\begin{array}{l}0 . \\
0 \\
3\end{array}$ & $\begin{array}{l}0 . \\
0 \\
2\end{array}$ & $\begin{array}{l}0 . \\
0 \\
9\end{array}$ & $\begin{array}{l}0 . \\
4 \\
6\end{array}$ & $\begin{array}{l}2 . \\
7 \\
2\end{array}$ \\
\hline $\begin{array}{l}1996 \\
-200 \\
5\end{array}$ & $\begin{array}{l}22 \\
.9 \\
5\end{array}$ & $\begin{array}{l}36 \\
.0 \\
7\end{array}$ & $\begin{array}{l}21 \\
.2 \\
4\end{array}$ & $\begin{array}{l}8 . \\
43\end{array}$ & $\begin{array}{l}5 . \\
0 \\
5\end{array}$ & $\begin{array}{l}1 . \\
8 \\
5\end{array}$ & $\begin{array}{l}0 . \\
7 \\
5\end{array}$ & $\begin{array}{l}0 . \\
0 \\
4\end{array}$ & $\begin{array}{l}0 . \\
0 \\
1\end{array}$ & $\begin{array}{l}0 . \\
0 \\
3\end{array}$ & $\begin{array}{l}0 . \\
5 \\
3\end{array}$ & $\begin{array}{l}3 . \\
0 \\
5\end{array}$ \\
\hline $\begin{array}{l}2006 \\
-201 \\
7\end{array}$ & $\begin{array}{l}28 \\
.0 \\
3\end{array}$ & $\begin{array}{l}26 \\
.1 \\
0\end{array}$ & $\begin{array}{l}23 \\
.7 \\
5\end{array}$ & $\begin{array}{l}10 \\
.6 \\
0\end{array}$ & $\begin{array}{l}5 . \\
2 \\
7\end{array}$ & $\begin{array}{l}1 . \\
5 \\
6\end{array}$ & $\begin{array}{l}0 . \\
1 \\
2\end{array}$ & $\begin{array}{l}0 . \\
0 \\
2\end{array}$ & $\begin{array}{l}0 . \\
0 \\
4\end{array}$ & $\begin{array}{l}0 . \\
5 \\
2\end{array}$ & $\begin{array}{l}0 . \\
4 \\
4\end{array}$ & $\begin{array}{l}3 . \\
5 \\
4\end{array}$ \\
\hline \multicolumn{13}{|c|}{ Sitanadi } \\
\hline $\begin{array}{l}1976 \\
-85\end{array}$ & $\begin{array}{l}25 \\
.4 \\
0\end{array}$ & $\begin{array}{l}34 \\
.8 \\
6\end{array}$ & $\begin{array}{l}22 \\
.8 \\
7\end{array}$ & $\begin{array}{l}7 . \\
69\end{array}$ & $\begin{array}{l}3 . \\
8 \\
2\end{array}$ & $\begin{array}{l}1 . \\
5 \\
8\end{array}$ & $\begin{array}{l}0 . \\
1 \\
0\end{array}$ & $\begin{array}{l}0 . \\
0 \\
1\end{array}$ & $\begin{array}{l}0 . \\
0 \\
1\end{array}$ & $\begin{array}{l}0 . \\
0 \\
3\end{array}$ & $\begin{array}{l}0 . \\
3 \\
9\end{array}$ & $\begin{array}{l}3 . \\
4 \\
6\end{array}$ \\
\hline $\begin{array}{l}1986 \\
-95\end{array}$ & $\begin{array}{l}26 \\
.9 \\
1\end{array}$ & $\begin{array}{l}26 \\
.4 \\
0\end{array}$ & $\begin{array}{l}28 \\
.6 \\
1\end{array}$ & $\begin{array}{l}7 . \\
98\end{array}$ & $\begin{array}{l}4 . \\
9 \\
5\end{array}$ & $\begin{array}{l}1 . \\
7 \\
2\end{array}$ & $\begin{array}{l}0 . \\
1 \\
9\end{array}$ & $\begin{array}{l}0 . \\
0 \\
3\end{array}$ & $\begin{array}{l}0 . \\
0 \\
2\end{array}$ & $\begin{array}{l}0 . \\
0 \\
9\end{array}$ & $\begin{array}{l}0 . \\
4 \\
8\end{array}$ & $\begin{array}{l}2 . \\
7 \\
3\end{array}$ \\
\hline $\begin{array}{l}1996 \\
-200 \\
5\end{array}$ & $\begin{array}{l}23 \\
.0 \\
3\end{array}$ & $\begin{array}{l}35 \\
.9 \\
3\end{array}$ & $\begin{array}{l}21 \\
.1 \\
6\end{array}$ & $\begin{array}{l}8 . \\
49\end{array}$ & $\begin{array}{l}5 . \\
0 \\
6\end{array}$ & $\begin{array}{l}1 . \\
8 \\
0\end{array}$ & $\begin{array}{l}0 . \\
7 \\
6\end{array}$ & $\begin{array}{l}0 . \\
0 \\
4\end{array}$ & $\begin{array}{l}0 . \\
0 \\
1\end{array}$ & $\begin{array}{l}0 . \\
0 \\
3\end{array}$ & $\begin{array}{l}0 . \\
5 \\
8\end{array}$ & $\begin{array}{l}3 . \\
1 \\
3\end{array}$ \\
\hline $\begin{array}{l}2006 \\
-201 \\
7\end{array}$ & $\begin{array}{l}27 \\
.7 \\
9\end{array}$ & $\begin{array}{l}26 \\
.5 \\
7\end{array}$ & $\begin{array}{l}23 \\
.5 \\
5\end{array}$ & $\begin{array}{l}10 \\
.6 \\
5\end{array}$ & $\begin{array}{l}5 . \\
3 \\
3\end{array}$ & $\begin{array}{l}1 . \\
5 \\
1\end{array}$ & $\begin{array}{l}0 . \\
1 \\
2\end{array}$ & $\begin{array}{l}0 . \\
0 \\
2\end{array}$ & $\begin{array}{l}0 . \\
0 \\
4\end{array}$ & $\begin{array}{l}0 . \\
5 \\
3\end{array}$ & $\begin{array}{l}0 . \\
3 \\
9\end{array}$ & $\begin{array}{l}3 . \\
5 \\
1\end{array}$ \\
\hline \multicolumn{13}{|c|}{ Agumbe } \\
\hline $\begin{array}{l}1976 \\
-85\end{array}$ & $\begin{array}{l}25 \\
.3 \\
6\end{array}$ & $\begin{array}{l}34 \\
.7 \\
9\end{array}$ & $\begin{array}{l}22 \\
.9 \\
7\end{array}$ & $\begin{array}{l}7 . \\
71\end{array}$ & $\begin{array}{l}3 . \\
8 \\
1\end{array}$ & $\begin{array}{l}1 . \\
5 \\
9\end{array}$ & $\begin{array}{l}0 . \\
1 \\
0\end{array}$ & $\begin{array}{l}0 . \\
0 \\
1\end{array}$ & $\begin{array}{l}0 . \\
0 \\
1\end{array}$ & $\begin{array}{l}0 . \\
0 \\
3\end{array}$ & $\begin{array}{l}0 . \\
3 \\
9\end{array}$ & $\begin{array}{l}3 . \\
4 \\
5\end{array}$ \\
\hline $\begin{array}{l}1986 \\
-95\end{array}$ & $\begin{array}{l}26 \\
.9 \\
6\end{array}$ & $\begin{array}{l}26 \\
.3 \\
3\end{array}$ & $\begin{array}{l}28 \\
.6 \\
2\end{array}$ & $\begin{array}{l}7 . \\
94\end{array}$ & $\begin{array}{l}5 . \\
0 \\
1\end{array}$ & $\begin{array}{l}1 . \\
7 \\
1\end{array}$ & $\begin{array}{l}0 . \\
1 \\
9\end{array}$ & $\begin{array}{l}0 . \\
0 \\
3\end{array}$ & $\begin{array}{l}0 . \\
0 \\
2\end{array}$ & $\begin{array}{l}0 . \\
0 \\
9\end{array}$ & $\begin{array}{l}0 . \\
5 \\
0\end{array}$ & $\begin{array}{l}2 . \\
7 \\
2\end{array}$ \\
\hline $\begin{array}{l}1996 \\
-200 \\
5\end{array}$ & $\begin{array}{l}23 \\
.1 \\
6\end{array}$ & $\begin{array}{l}35 \\
.7 \\
5\end{array}$ & $\begin{array}{l}21 \\
.0 \\
4\end{array}$ & $\begin{array}{l}8 . \\
53\end{array}$ & $\begin{array}{l}5 . \\
0 \\
9\end{array}$ & $\begin{array}{l}1 . \\
7 \\
5\end{array}$ & $\begin{array}{l}0 . \\
7 \\
6\end{array}$ & $\begin{array}{l}0 . \\
0 \\
4\end{array}$ & $\begin{array}{l}0 . \\
0 \\
1\end{array}$ & $\begin{array}{l}0 . \\
0 \\
3\end{array}$ & $\begin{array}{l}0 . \\
5 \\
8\end{array}$ & $\begin{array}{l}3 . \\
2 \\
5\end{array}$ \\
\hline $\begin{array}{l}2006 \\
-201 \\
7\end{array}$ & $\begin{array}{l}27 \\
.7 \\
6\end{array}$ & $\begin{array}{l}26 \\
.5 \\
1\end{array}$ & $\begin{array}{l}23 \\
.7 \\
1\end{array}$ & $\begin{array}{l}10 \\
.6 \\
9\end{array}$ & $\begin{array}{l}5 . \\
3 \\
6\end{array}$ & $\begin{array}{l}1 . \\
5 \\
0\end{array}$ & $\begin{array}{l}0 . \\
1 \\
3\end{array}$ & $\begin{array}{l}0 . \\
0 \\
2\end{array}$ & $\begin{array}{l}0 . \\
0 \\
4\end{array}$ & $\begin{array}{l}0 . \\
5 \\
3\end{array}$ & $\begin{array}{l}0 . \\
3 \\
3\end{array}$ & $\begin{array}{l}3 . \\
4 \\
1\end{array}$ \\
\hline
\end{tabular}

Table.10 Standardized precipitation index (SPI) (McKee et. al., 1993)

\begin{tabular}{cc}
\hline SPI Values & Category \\
\hline 2.00 and more & Extremely wet \\
1.50 to 1.99 & Severely wet \\
1.00 to 1.49 & Moderately wet \\
0 to 0.99 & Mildly wet \\
0 to- 0.99 & Mild drought \\
-1.00 to -1.49 & Moderately drought \\
-1.50 to-1.99 & Severe drought \\
-2 and less & Extreme drought
\end{tabular}

Table.11 Standardized precipitation index (SPI) of rain gauge stations in Sitanadi basin 


\begin{tabular}{|c|c|c|c|c|c|c|c|c|c|c|c|c|c|}
\hline Year & $\begin{array}{c}\text { Brahmavar } \\
\text { a }\end{array}$ & $\begin{array}{c}\text { Kot } \\
\mathbf{a}\end{array}$ & $\begin{array}{l}\text { Halad } \\
\quad \mathbf{i}\end{array}$ & $\begin{array}{c}\text { Ardi } \\
\text { (Albadi } \\
\text { ) }\end{array}$ & $\underset{\mathbf{i}}{\text { Sitanad }}$ & $\begin{array}{c}\text { Agumb } \\
\text { e }\end{array}$ & $\begin{array}{l}09-1 \\
0 \\
10-1\end{array}$ & 0.48 & $\begin{array}{l}0.93 \\
-0.2\end{array}$ & -0.21 & 0.13 & -0.89 & -0.35 \\
\hline $76-7$ & & & & & & & 1 & -0.44 & 7 & -0.76 & -1.11 & -1.81 & -0.54 \\
\hline 7 & 1.12 & 0.97 & 0.44 & 1.11 & 0.90 & 0.10 & $11-1$ & & & & & & \\
\hline $77-7$ & & & & & & & 2 & -0.38 & 0.50 & -1.06 & -0.55 & -1.59 & -1.20 \\
\hline 8 & 1.08 & 1.26 & 3.96 & 0.89 & 0.77 & -0.06 & $12-1$ & & -0.3 & & & & \\
\hline $78-7$ & & & & & & & 3 & -0.06 & 6 & -0.37 & -0.45 & -1.40 & -0.32 \\
\hline 9 & 2.43 & 1.30 & 2.05 & 2.10 & 0.99 & -0.09 & $13-1$ & & -0.9 & & & & \\
\hline $79-8$ & & & & & & & 4 & -1.30 & 9 & -0.35 & -0.12 & -0.43 & -1.18 \\
\hline 0 & 1.48 & 1.70 & 0.91 & 0.32 & 0.46 & -0.83 & $14-1$ & & -2.4 & & & & \\
\hline $80-8$ & & -0.1 & & & & & 5 & -0.29 & 5 & -0.59 & 0.55 & -0.17 & -1.55 \\
\hline 1 & -0.30 & 3 & -0.75 & -1.74 & -1.67 & -1.54 & $15-1$ & & & & & & \\
\hline $81-8$ & & -0.9 & & & & & 6 & 0.26 & 1.06 & 1.78 & 1.11 & -0.69 & -1.19 \\
\hline 2 & 0.11 & 4 & -0.64 & -0.97 & -1.12 & -0.02 & $16-1$ & & -0.4 & & & & \\
\hline $82-8$ & & & & & & & 7 & -0.73 & 7 & -1.31 & -0.15 & -0.46 & -1.20 \\
\hline 3 & 0.84 & 0.02 & -1.18 & -0.27 & 0.33 & -0.05 & & & & & & & \\
\hline $\begin{array}{l}83-8 \\
4\end{array}$ & 1.85 & 0.69 & -0.03 & 1.13 & -0.54 & 1.29 & & Table.12 & Numbe & $r$ and pe & arcent of & years & \\
\hline $\begin{array}{l}84-8 \\
5 \\
85-8\end{array}$ & -0.14 & $\begin{array}{c}-0.8 \\
7\end{array}$ & -0.89 & -1.47 & -0.84 & -1.04 & Class & $\begin{array}{l}\text { Brahmav } \\
\text { ara }\end{array}$ & Kota & Haladi & $\begin{array}{l}\text { Ardi } \\
\text { (Albad } \\
\text { i) } \\
\end{array}$ & $\begin{array}{l}\text { Sitan } \\
\text { adi }\end{array}$ & $\begin{array}{l}\text { Agum } \\
\text { be }\end{array}$ \\
\hline $\begin{array}{l}\text { ly-o } \\
6 \\
86-8\end{array}$ & 1.14 & $\begin{array}{l}0.00 \\
-0.5\end{array}$ & -1.04 & -0.68 & 0.58 & 0.68 & $\begin{array}{l}\text { Extreme } \\
\text { ly wet }\end{array}$ & $1(2.38)$ & Nil & $2(4.76)$ & $2(4.76)$ & )$^{1(2.38}$ & $\begin{array}{l}2(4.76 \\
)\end{array}$ \\
\hline $\begin{array}{l}86-8 \\
7 \\
87-8\end{array}$ & 0.86 & $\begin{array}{c}-0.5 \\
8 \\
-0.9\end{array}$ & 0.20 & 0.78 & 1.58 & 2.14 & $\begin{array}{l}\text { Severely } \\
\text { wet }\end{array}$ & $2(4.76)$ & $\begin{array}{l}2 \\
(4.76)\end{array}$ & $2(4.76)$ & $1(2.38)$ & $\begin{array}{l}3(7.14 \\
)\end{array}$ & $\begin{array}{l}1(2.38 \\
)\end{array}$ \\
\hline $\begin{array}{l}8 \\
88-8\end{array}$ & 0.21 & $\begin{array}{c}4 \\
-0.0\end{array}$ & -0.62 & -0.82 & -0.62 & 1.09 & $\begin{array}{l}\text { Moderat } \\
\text { ely wet }\end{array}$ & $5(11.90)$ & $\begin{array}{l}6 \\
(14.28)\end{array}$ & $1(2.38)$ & $\begin{array}{l}4 \\
(9.52)\end{array}$ & $\begin{array}{l}2(4.76 \\
)\end{array}$ & $\begin{array}{l}5 \\
(11.9)\end{array}$ \\
\hline $\begin{array}{l}88-8 \\
9 \\
89-9\end{array}$ & 1.87 & $\begin{array}{c}-0.0 \\
1\end{array}$ & 0.41 & 0.54 & 1.43 & -0.81 & $\begin{array}{l}\text { Mildly } \\
\text { wet }\end{array}$ & $11(26.19)$ & $\begin{array}{l}12(28 . \\
57)\end{array}$ & $\begin{array}{l}9(21.4 \\
2)\end{array}$ & $\begin{array}{l}11(26 . \\
19)\end{array}$ & $\begin{array}{l}15 \\
(35.7)\end{array}$ & $\begin{array}{l}8 \\
(19.0)\end{array}$ \\
\hline $\begin{array}{l}0 \\
90-9\end{array}$ & 0.81 & 1.09 & 0.71 & 0.73 & 1.29 & 0.70 & $\begin{array}{l}\text { Mild } \\
\text { drought }\end{array}$ & $17(40.47)$ & $\begin{array}{l}18(42 . \\
85)\end{array}$ & $\begin{array}{l}24(57 . \\
14)\end{array}$ & $\begin{array}{l}18 \\
(42.8)\end{array}$ & $\begin{array}{l}14 \\
(33.3)\end{array}$ & $\begin{array}{l}14(33 . \\
3)\end{array}$ \\
\hline $\begin{array}{l}1 \\
91-9\end{array}$ & 0.54 & 1.46 & 0.15 & 0.07 & 0.18 & 2.30 & $\begin{array}{l}\text { Moderat } \\
\text { ely } \\
\text { drought }\end{array}$ & $3(7.14)$ & $\begin{array}{l}2 \\
(4.76)\end{array}$ & $4(9.52)$ & $4(9.52)$ & $\begin{array}{l}4(9.52 \\
)\end{array}$ & $\begin{array}{l}7(16.7 \\
)\end{array}$ \\
\hline $\begin{array}{l}2 \\
92-9\end{array}$ & 0.81 & $\begin{array}{l}1.43 \\
-0.8\end{array}$ & 0.32 & 0.55 & 0.27 & 0.31 & $\begin{array}{l}\text { Severe } \\
\text { drought }\end{array}$ & $3(7.14)$ & $\begin{array}{l}1 \\
(2.38)\end{array}$ & Nil & $1(2.38)$ & $\begin{array}{l}3(7.14 \\
)\end{array}$ & $\begin{array}{l}2(4.76 \\
)\end{array}$ \\
\hline $\begin{array}{l}3 \\
93-9 \\
4\end{array}$ & -1.63 & $\begin{array}{c}1 \\
-1.2 \\
9\end{array}$ & -0.26 & -0.03 & -0.31 & -0.05 & $\begin{array}{l}\text { Extreme } \\
\text { drought }\end{array}$ & NIL & $1(2.38)$ & Nil & $1(2.38)$ & Nil & Nil \\
\hline
\end{tabular}

\section{H. Standardized Precipitation Index (SPI)}

As Lloyd-Hughes \& Saunders (2002) mentioned, the SPI is computed by fitting a probability density function to the frequency distribution of precipitation summed over the time scale of interest. Each probability density function is then transformed into the standardized normal distribution with a mean of 0 and standard deviations of 1 (Eq. 1.) Thus, the SPI is said to be normalized in location and time scale. Once standardized, the strength of the anomaly is classified as set out in Table.10. Equation SPI=X-X1/ $\sigma$, where $\mathrm{X}$ is the annual rainfall of any year, $\mathrm{X} 1$ is the Mean annual rainfall over the years, and $\sigma$ Standard deviation of the rainfall series. From the rainfall data of 42 years, SPI was calculated for the whole period and presented in the Table.11. SPI values of the rain gauge stations of the Sitanadi basin varies from +2.43 to -1.82 in Brahmavara, from +1.7 to -2.45 in Kota, from +3.96 to -1.31 in Haladi, from +2.58 to -2.50 in Ardi, from +2.02 to -1.81 in Sitanadi and from +2.3 to -1.55 in Agumbe. Table. 12 shows the number and percent of years in different category of rainfall. 


\section{CONCLUSION}

In this study, precipitation data with longer recording histories were used to calculate the standardized precipitation index values for Sitanadi basin. Result shows that Kota and Ardi $(2.38 \%)$ stations experience extreme drought. Severe drought cases are observed in all the rain gauge stations except Haladi. Percent of severe drought varies from $7.14 \%$ in Brahmavara and Sitanadi and $2.38 \%$ in Kota and Ardi stations. In Haladi, Sitanadi and Agumbe there were extreme drought periods during the study period. An extremely wet year percentage varies from $4.76 \%$ in Haladi, Ardi and Agumbe to $2.38 \%$ in Brahmavara and Sitanadi. In Kota no extreme wet period is recorded. Sitanadi recorded highest severely wet period (7.14\%) and Ardi and Agumbe recorded $(2.38 \%)$ minimum severely wet period. Higher abnormality is observed in PRM season compared to POM season in all rain gauge stations. The validation based on station gauge rainfall data showed that the SPIs calculated were reliable for monitoring drought regions. The analyses of drought showed that the frequency of different intensity droughts presented significant spatial heterogeneity in each rain gauge region.

\section{REFERENCES}

1. Al-Obaidy A (2012) Estimation of Impacts of Rainfall on Cereal Production in the Northern Region of Iraq for the Period 1992-2008. Bus Econ Res.

2. Al-Qinna MI, Hammouri NA, Obeidat MM, Ahmed F (2011) Drought analysis in Jordan under current and future climates. Clim Change 106:421-440.

3. Halwatura D, Lechner AM, Arnold S (2015) Drought severity-duration-frequency curves: a foundation for risk assessment and planning tool for ecosystem establishment in post-mining landscapes. Hydrol Earth Syst Sci 19(2):1069-1091.

4. Hao ZC, AghaKouchak A (2013) Multivariate standardized drought index: a parametric multi-index model. Adv Water Resour 57:12-18.

5. Global Information and Early Warning System on Food and Agriculture (GIEWS) (2014) Serious food security concerns following escalation of conflict. Special Alert No. 332. IRAQ.

6. Liu L, Hong Y, Bednarczyk CN, Yong B, Shafer MA, Riley R, Hocker JE (2012) Hydro-Climatological drought analyses and projections using meteorological and hydrological drought indices: a case study in blue river basin, Oklahoma. Water Resour Manag 26(10):2761-2779.

7. Lloyd-Hughes B (2014) The impracticality of a universal drought definition. Theor Appl Climatol 117:607-611.

8. Lorenzo-Lacruz J, Morán-Tejeda E (2016) Spatio-temporal patterns of meteorological droughts in the Balearic Islands (Spain). Cuadernos de Investigación Geográfica.

9. Zhang, M.; He, J.; Wang, B.; Wang, S.; Li, S.; Liu, W.; Ma, X. Extreme drought changes in Southwest China from 1960 to 2009. J. Geogr. Sci. 2013, 23, 3-16.

10. Yu, M.; Li, Q.; Hayes, M.J.; Svoboda, M.D.; Heim, R.R. Are droughts becoming more frequent or severe in China based on the standardized precipitation evapotranspiration index: 1951-2010. Int. J. Climatol. 2014, 34, 545-558.

11. Tao, H.; Fischer, T.; Zeng, Y.; Fraedrich, K. Evaluation of TRMM 3B43 precipitation data for drought monitoring in Jiangsu Province, China. Water 2016, 8, 221

12. Zeng, H.; Li, L.; Li, J. The evaluation of TRMM Multisatellite Precipitation Analysis (TMPA) in drought monitoring in the Lancang River Basin. J. Geogr. Sci. 2012, 22, 273-282. 